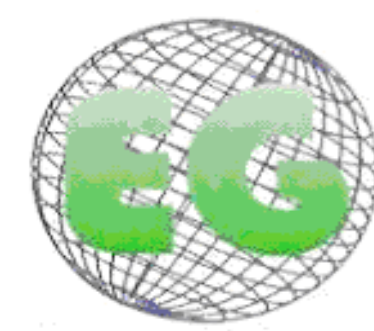

ISSN 1695-6141

$N^{\circ} 23$
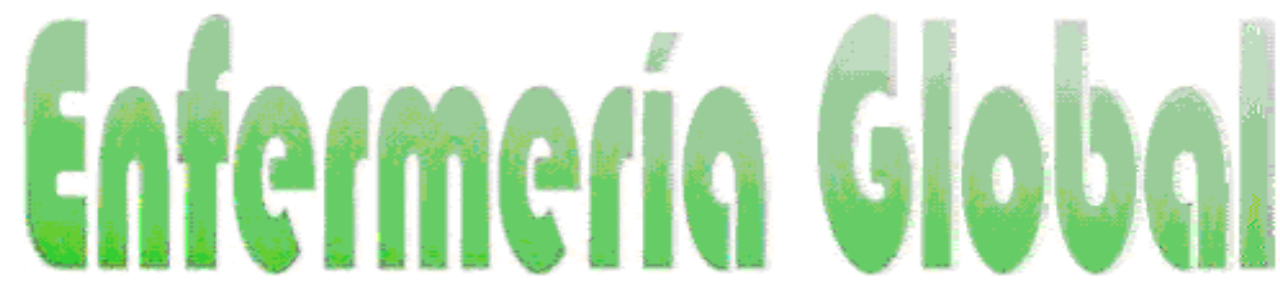

Revista electrónica trimestral de Enfermería

www.um.eslegloball

\title{
La no realización del autoexamen de mama en Tunja, Colombia
}

Non realisation of breast self-examination in Tunja, Colombia

\author{
*Ospina Díaz, JM., "*Manrique Abril, FG., "**Veja, NA., "**Morales Pacheco, AL., \\ ${ }^{* * *}$ Ariza R., NE. \\ *MD. MSc. Epidemiología. Profesor Asociado UPTC. **RN. PhD. Profesor Asociado UPTC, Universidad \\ Nacional de Colombia. ***Enfermera. Grupo de Investigación en Salud Pública. Universidad Pedagógica y \\ Tecnológica de Colombia Tunja - Boyacá. Colombia.
}

Palabras clave: Promoción de la salud; Prevención primaria; Autoexamen de mamas; Neoplasias de la mama. Keywords: Health promotion; Primary prevention; Breast self-examination; Breast neoplasm (source: MeSH, NLM).

\section{RESUMEN}

Objetivo. Identificar factores socioculturales y psicosociales determinantes de no realización del auto examen de mamas (AEM) en mujeres residentes en Tunja, Colombia.

Materiales y métodos. Un estudio anidado de casos y controles se llevó a cabo a partir de 218 casos y 225 controles. La muestra se seleccionó mediante muestreo aleatorio estratificado. Se evaluó sobre los factores socioculturales, el conocimiento y la práctica del AEM.

Resultados. Edad promedio 32,9 años (SD=9,44); 58,7\% con pareja estable; $42,4 \%$ trabaja; $25,6 \%$ secundaria incompleta; $23,5 \%$ fuma; $21,5 \%$ planifica con métodos hormonales; $10,6 \%$ tiene antecedentes familiares de carcinoma mamario; 10,2\% asiste a programas de promoción y prevención; 7,2\% antecedente de enfermedad mamaria. Los factores que se encontraron significativamente asociados con la no realización del AEM son: no haber recibido información $(\mathrm{OR}=14,08)$; no asistir a programas de prevención del cáncer de mamá $(O R=9,21)$; bajo nivel de conocimientos sobre cáncer de mama $(\mathrm{OR}=8,96)$; no considerar importante $\mathrm{AEM}(\mathrm{OR}=8,71)$; no contar con apoyo familiar $(\mathrm{OR}=3,18)$; no reconoce la lactancia como factor protector en cáncer de mama $(\mathrm{OR}=2,46)$; no realizar actividad física $(\mathrm{OR}=2,38)$; no haber terminado la secundaria $(\mathrm{OR}=1,94)$; no uso de anticoncepción $(\mathrm{OR}=2,27)$; no ha consultado sobre $A E M(O R=1,97)$; régimen subsidiado o vinculado (OR=1,77); uso de métodos de planificación menor de 3 años $(\mathrm{OR}=1,94)$; no tener antecedente de aborto (OR=1,92); no trabajar (OR=1,47).

Conclusión. Se encuentran factores de riesgo, susceptibles de intervención educativa, que podrían mejorar sustancialmente la cobertura de los programas de $\mathrm{P}$ y $\mathrm{P}$ y la adherencia a la práctica del AEM. 


\section{ABSTRACT}

Objective. To identify sociocultural and psychosocial factors that determine non realisation of Breast Self-Examination (BSE) in women resident in Tunja, Colombia.

Materials and methods. A nested case-control study was carried with 218 cases and 225 controls. The sample was selected by statified random sample. Assessment was made of sociocultural factors, knowledge and BSE practice.

Results. Mean age 32.9 years (SD = 9.44), 58.7\% with steady partner, $42.4 \%$ work, $25.6 \%$ incomplete secondary, $23.5 \%$ smokers, $21.5 \%$ uses hormonal methods, $10.6 \%$ have a family history of breast carcinoma, $10.2 \%$ attend promotion and prevention programs, $7.2 \%$ have history of breast disease. The factors that were significantly associated with non-practice of the BSE were not having received information about $B S E(O R=14.08)$; non attendance at Promotion and Prevention programs $(\mathrm{OR}=9.21)$, low levels of knowledge about breast cancer $(\mathrm{OR}=8.96)$, BSE was not considered important $(O R=8.71)$; not having family support $(O R=3.18)$; not recognizing breastfeeding as a protective factor in breast cancer $(\mathrm{OR}=2.46)$, no physical activity $(\mathrm{OR}=2.38)$ not completed high school (OR $=1.94)$, not using contraception ( $O R=2.27)$; not attending the review consultation BSE $(\mathrm{OR}=1.97)$ subsidized or linked health-regime $(\mathrm{OR}=1.77)$, less than 3 years use of planning methods $(\mathrm{OR}=1.94)$, no record of abortion $(\mathrm{OR}=1.92)$, not in work $(\mathrm{OR}=1,47)$.

Conclusion. We found risk factors that are susceptible to educational intervention which could substantially improve the cover of Health Promotion and Disease Prevention programs and adherence to the practice of BSE.

\section{INTRODUCCIÓN}

Hasta hace unos pocos años, en Colombia el cáncer de mama no era considerado un problema significativo de salud, debido a que los menguados recursos se enfocaban preferencialmente a la atención de la morbimortalidad ocasionada por lesiones de causa externa y enfermedades infecciosas, que se reconocían como los principales problemas de salud pública. Sin embargo, inevitablemente, se ocasionaban gastos significativos en atención del cáncer, el cual por regla general se abordaba en los hospitales en estadíos tardíos. En la actualidad, el cáncer como enfermedad, reclama cada vez más preparación y mejor atención en la medida que el ambiente sanitario y socio económico cambia y la esperanza de vida se alarga. ${ }^{(1)}$

En Colombia, la mortalidad por cáncer de mama muestra tendencia al incremento en la última década ${ }^{(2)}$. Para el año 2000, el cáncer de mama ocupó el tercer lugar como causa de muerte por cáncer entre mujeres, con 1542 muertes registradas, después del cáncer de estómago y el de cuello uterino ${ }^{(2)}$. En el país no se dispone de información sobre los estadíos en que se diagnostica la enfermedad; los datos de las pacientes atendidas en año 2004, en el Instituto Nacional de Cancerología (INC), muestran que alrededor del $64 \%$ de los casos nuevos llegan en estadios avanzados de II B y más ${ }^{(3)}$.

El cáncer de mama es considerado un problema prioritario de salud pública a nivel mundial, ya que se le reconoce como primera causa de muerte por cáncer entre las mujeres ${ }^{(4)}$. La frecuencia de presentación ha aumentado gradualmente, probablemente por factores como las gestaciones precoces, el uso de hormonas y los cambios en la actividad física ${ }^{(5)}$. En los países en desarrollo la incidencia es significativamente menor que en los países desarrollados ${ }^{(6)}$. Además, en razón a que el diagnóstico es más tardío en los países pobres, se encuentra que la supervivencia es también menor ${ }^{(7)}$. 
Un problema de capital importancia en el diagnóstico oportuno tiene que ver con la casi total ausencia de signos o síntomas clínicos en los estadíos iniciales de la historia natural de esta enfermedad, factor que se acentúa en las mujeres mayores y de nivel social y educativo más bajo $^{(8)}$.

La complejidad de factores que determinan la patogenia del cáncer de mama limita de momento las posibilidades de la prevención primaria. Por esta razón se considera primordial enfatizar en los mecanismos que permitan realizar el diagnóstico precoz, por cuanto se ha demostrado que la supervivencia de las mujeres diagnosticados en estadíos tempranos es 2,5 veces superior comparada con la supervivencia de mujeres a quienes se diagnóstica en estadios tardíos ${ }^{(9)}$.

Diversos estudios han demostrado la importancia de factores sociodemográficos en relación con la ocurrencia del diagnóstico tardío entre los que se ha destacado la accesibilidad a los servicios de salud y las características de calidad en las que se desarrolla la educación y promoción de participación en las acciones de fomento y prevención ${ }^{(7,10)}$. En los países subdesarrollados y en ciertos grupos étnicos de los países desarrollados. El análisis de factores socioculturales de las razones por las que no se llega a un diagnóstico precoz del cáncer de mama ha llevado a vislumbrar otros determinantes importantes, entre los que se encuentran la noción de apoyo familiar y social que eventualmente mejoran la motivación y el interés de las mujeres para realizar el autoexamen de mama y acudir a los servicios de salud con mayor frecuencia ${ }^{(11)}$.

Los significativos aumentos en la incidencia, así como la evidencia que demuestra la mejoría en la supervivencia y el pronóstico, en la medida que el diagnóstico se haga más temprano, lleva a que este estudio se proponga explorar determinantes sociales, económicas y culturales relacionadas con la cobertura y adherencia a la práctica del autoexamen de mama, susceptibles de modificación a partir de intervenciones adecuadas de los agentes de salud.

\section{MATERIAL Y MÉTODO}

El presente trabajo se diseñó y ensambló como un estudio observacional de casos y controles, anidado. De la población censada en un análisis previo poblacional se seleccionó una muestra de 218 mujeres que no practican el AEM (casos) y 225 que manifestaron sí realizarlo (controles); previo consentimiento informado, a todas se les aplicó mediante entrevista directa un cuestionario diseñado con anterioridad y validado mediante prueba piloto por el grupo de investigación, el cual consta de 45 preguntas que exploran aspectos sociodemográficos, hábitos de comportamiento, aspectos de la vida reproductiva de la mujer y conocimientos, actitudes y prácticas sobre el autoexamen de mama.

La muestra fue estimada en el programa STATCALC de Epi-info $2002^{\circledR}$ para evaluar con un $95 \%$ de confiabilidad, diferencias del $5 \%$ o más en el grado de factores asociados en la no realización del auto examen de mama.

Mediante muestreo multietápico por áreas geográficas, que incluyó las ocho zonas urbanas de la ciudad de Tunja, clasificadas en el Plan de Ordenamiento Territorial (POT) ${ }^{(12)}$, con los respectivos barrios y número de viviendas, se cuantificó el tamaño de muestra adecuado y al azar se procedió a seleccionar a las personas a entrevistar por zona y barrio. Se definió como unidad de observación y análisis a las mujeres mayores de 18 años, que cumplían los criterios de inclusión y aceptaron libremente participar en el estudio. 
Los cuestionarios fueron desarrollados por profesionales previamente entrenados, mediante entrevista directa. Las personas seleccionadas fueron visitadas en sus casas, se les informó de los objetivos del estudio, se les garantizó su privacidad y se obtuvo su anuencia mediante la firma del consentimiento informado. La sistematización de los datos se realizó por duplicado y se validó para detectar y corregir posibles errores de digitación en el programa VALIDATE Epilnfo v 6-04d (CDC/Epilnfo, versión 6.04d).

El análisis univariado permitió examinar las características y comparabilidad de los grupos, mediante la estimación de medias para variables continuas, proporciones para las variables categóricas o nominales; todas reportadas con sus correspondientes intervalos de confianza (IC) a un nivel de significancia de 0.05 .

Posteriormente se adelantó análisis exploratorio de la asociación de las variables explicatorias incluidas, con la variable de salida. Esto fue realizado mediante la estimación de la Odds Ratio (OR) y sus respectivos IC al 95\%, junto con el valor p en la prueba exacta de Fisher. Un análisis estratificado fue realizado de manera exploratoria, con el fin de identificar posibles variables confusoras.

\section{RESULTADOS}

\section{Caracterización de la muestra}

El rango de edades osciló entre 20 y 59 años con una media de 32,95 (SD=9,4); fueron incluidas mujeres de 91 barrios ubicados en las 8 zonas definidas en el Plan de Ordenamiento Territorial (POT) para la ciudad de Tunja, de manera proporcional al volumen de población establecido en el Censo de 2005, lo que garantiza la representatividad de la muestra.

El 64,6\% se clasificó como adulta joven (menor de 50 años); 39,7\% registra sobrepeso u obesidad; $58,7 \%$ tiene pareja estable; $25,6 \%$ no ha terminado la educación secundaria; $88,7 \%$ presentó la menarquía antes de los 16 años, con edad promedio de 13,5 años $(\mathrm{SD}=1,6) ; 75,4 \%$ tiene al menos un hijo y de ellas $5 \%$ tuvo su primer parto después de los 30 años; $27,5 \%$ nunca ha lactado; $12,2 \%$ registra antecedente de aborto; $6,1 \%$ ya ha llegado a la menopausia y la edad promedio de ella es a los 48,8 años ( $\mathrm{SD}=3,36) ; 47,4 \%$ usa métodos de planificación familiar, con un tiempo promedio de 4,5 años (rango 1-20, SD=3,8); 7,2\% registra antecedente de enfermedad mamaria, principalmente mastitis $(14=3,2 \%)$ y enfermedad fibroquística (13=2,9\%); 10,6 manifiesta tener antecedente familiar de cáncer de mama; $23,5 \%$ fuma y $27,3 \%$ consume alcohol; 34,5\% habitualmente desarrolla algún tipo de actividad física. En la Tabla 1 se muestra la comparabilidad de los dos grupos.

Tabla 1. Comparabilidad de los grupos

\begin{tabular}{|l|c|c|c|}
\hline \multicolumn{1}{|c|}{ VARIABLE } & CASO & CONTROL & P \\
\hline Promedio de edad (SD) & $32,55(9,3)$ & $33,35(9,57)$ & 0,37 \\
\hline IMC normal (\%) & $120(55.0)$ & $138(61,3)$ & 0,21 \\
\hline Estrato Socio-Económico 3 o menor (\%) & $203(93,1)$ & $214(95,1)$ & 0,49 \\
\hline Con pareja estable (\%) & $129(49,6)$ & $131(50,4)$ & 0,91 \\
\hline
\end{tabular}




\begin{tabular}{|l|c|c|c|}
\hline Trabaja fuera de casa (\%) & $82(37,6)$ & $106(47,1)$ & 0,054 \\
\hline Secundaria completa o más (\%) & $148(67,8)$ & $181(80,4)$ & 0,0036 \\
\hline Afiliación al SGSS Contributivo (\%) & $103(47,2)$ & $138(61,3)$ & 0,004 \\
\hline Promedio edad menarquía (SD) & $13,4(1,7)$ & $13,59(1,66)$ & 0,23 \\
\hline Promedio de hijos (SD) & $1,87(1,54)$ & $1,65(1,46)$ & 0,12 \\
\hline Promedio de edad 1er. Parto (SD) & $22,27(4,7)$ & $22,36(4,4)$ & 0,83 \\
\hline Realizó Lactancia (\%) & $160(49,7)$ & $162(50,3)$ & 0,82 \\
\hline Lactancias > meses (\%) & $143(50,9)$ & $138(49,1)$ & 0,4 \\
\hline Antecedente de Aborto (\%) & $19(8,7)$ & $35(15,6)$ & 0,039 \\
\hline Menopausia (\%) & $12(5,5)$ & $15(6,7)$ & 0,75 \\
\hline Usa Métodos de PF (\%) & $81(37,2)$ & $129(57,3)$ & $<0,001$ \\
\hline Usa Hormonales (\%) & $38(18,4)$ & $57(25,4)$ & 0,056 \\
\hline Tiempo promedio uso MPF (SD) & $3,87(2,9)$ & $4,96(4,2)$ & 0,001 \\
\hline Antecedente de enfermedad de la mama (\%) & $12(5,5)$ & $20(8,9)$ & 0,23 \\
\hline Antecedente Familiar de Ca mamario (\%) & $18(8,3)$ & $29(12,9)$ & 0,15 \\
\hline Fuma (\%) & & & \\
\hline & & & \\
\hline
\end{tabular}

En la Tabla 2 se muestran asociaciones estadísticamente significativas entre la variable de salida "No realiza autoexamen de mama" y algunos factores que en la literatura se han reportado como asociados con ella. Algunos de estos factores reportaron OR mayor que 1 aunque la asociación no fue estadísticamente significativa, estas covariables fueron: mayor de 50 años $(\mathrm{OR}=1,36, \mathrm{p}=0,12)$; Sobrepeso u obesidad ( $\mathrm{OR}=1,22, \mathrm{p}=0,17)$; menarquía después de los 15 años ( $O R=1,39, p=0,27)$; tiene más de 3 hijos ( $O R=1,43, p=0,12$ ); primer hijo después de los 30 años ( $\mathrm{OR}=1,45, \mathrm{p}=0,30)$; fuma $(\mathrm{OR}=0,77, \mathrm{p}=0,24)$; más de 5 cigarrillos al día ( $\mathrm{OR}=1,43, \mathrm{p}=0,24)$; consume bebidas alcohólicas $(\mathrm{OR}=1,28, \mathrm{p}=0,24) ; 6$ o más copas/semana $(\mathrm{OR}=1,37, \mathrm{p}=0,24)$ 
Tabla 2. Asociación entre la variable de salida "No realiza autoexamen de mama" y algunas variables explicatorias.

\begin{tabular}{|c|c|c|c|c|c|c|}
\hline \multicolumn{2}{|l|}{ VARIABLE } & \multirow{2}{*}{$\begin{array}{r}\text { CASO } \\
\\
120 \\
\end{array}$} & \multirow{2}{*}{\begin{tabular}{|c} 
CONTROL \\
18
\end{tabular}} & \multirow{3}{*}{$\begin{array}{r}\text { OR } \\
14,08\end{array}$} & \multirow{3}{*}{$\begin{array}{r}\text { I.C.95\% } \\
8,1-24,4\end{array}$} & \multirow{3}{*}{$\begin{array}{c}\mathbf{p} \\
<0,0001\end{array}$} \\
\hline \multirow{2}{*}{ Ha recibido información sobre AEM } & NO & & & & & \\
\hline & SI & 98 & 207 & & & \\
\hline \multirow{2}{*}{$\begin{array}{l}\text { Asiste a Programas de promoción y } \\
\text { prevención }\end{array}$} & NO & 213 & 185 & \multirow{2}{*}{9,21} & \multirow{2}{*}{$3,5-23,8$} & \multirow{2}{*}{$<0,0001$} \\
\hline & SI & 5 & 40 & & & \\
\hline \multirow[b]{2}{*}{ Tiene conocimientos sobre Ca de mama } & $\mathrm{NO}$ & 134 & 34 & \multirow[b]{2}{*}{8,96} & \multirow[b]{2}{*}{$5,6-14,1$} & \multirow[b]{2}{*}{$<0,0001$} \\
\hline & SI & 84 & 191 & & & \\
\hline \multirow{3}{*}{ Considera importante realizar AEM } & NO & 53 & 8 & \multirow{3}{*}{8,71} & \multirow{3}{*}{$4,0-18,8$} & \multirow{3}{*}{$<0,0001$} \\
\hline & & & & & & \\
\hline & SI & 165 & 217 & & & \\
\hline \multirow[b]{2}{*}{ Existe apoyo familiar } & NO & 45 & 17 & \multirow[b]{2}{*}{3,18} & \multirow[b]{2}{*}{$1,7-5,76$} & \multirow[b]{2}{*}{$<0,0001$} \\
\hline & SI & 173 & 208 & & & \\
\hline \multirow{2}{*}{$\begin{array}{l}\text { Reconoce protección de la lactancia } \\
\text { para prevenir Ca de mama }\end{array}$} & NO & 211 & 208 & \multirow{2}{*}{2,46} & $1,08-$ & \multirow{2}{*}{0,0339} \\
\hline & SI & 7 & 17 & & & \\
\hline \multirow{3}{*}{ Practica regularmente actividad física } & NO & 164 & 126 & \multirow{3}{*}{2,38} & \multirow{3}{*}{$1,5-3,57$} & \multirow{3}{*}{$<0,0001$} \\
\hline & & & & & & \\
\hline & SI & 54 & 99 & & & \\
\hline \multirow{2}{*}{$\begin{array}{l}\text { Utiliza algún método de Planificación } \\
\text { familiar }\end{array}$} & NO & 137 & 96 & \multirow{2}{*}{2,27} & \multirow{2}{*}{$1,5-3,32$} & \multirow{2}{*}{$<0,0001$} \\
\hline & SI & 81 & 129 & & & \\
\hline Ha preguntado en la consulta sobre & $\mathrm{NO}$ & 125 & 91 & & & \\
\hline AFM & & 02 & 134 & 1,97 & $1,3-2,8$ & 0,0002 \\
\hline & $\mathrm{NO}$ & 70 & 44 & & & \\
\hline Terminó la Educación Secundaria & & & & 1,94 & $1,25-3,0$ & 0,0017 \\
\hline & SI & 148 & 181 & & & \\
\hline
\end{tabular}




\begin{tabular}{|c|c|c|c|c|c|c|}
\hline \multirow{2}{*}{ Ha usado MPF por tres años o más } & $\mathrm{NO}$ & 37 & 39 & \multirow{2}{*}{1,94} & \multirow{2}{*}{$1,09-3,9$} & \multirow{2}{*}{0,0172} \\
\hline & SI & 44 & 90 & & & \\
\hline \multirow{2}{*}{ Antecedente de haber tenido un aborto } & $\mathrm{NO}$ & 199 & 190 & \multirow{2}{*}{1,92} & \multirow{2}{*}{$1,0-3,49$} & \multirow{2}{*}{0,0194} \\
\hline & $\mathrm{SI}$ & 10 & 3 & & & \\
\hline \multirow{3}{*}{ Afiliada al Régimen Contributivo } & $\mathrm{NO}$ & 115 & 87 & \multirow{3}{*}{1,77} & \multirow{3}{*}{$1,21-2,5$} & \multirow{3}{*}{0,0019} \\
\hline & & & & & & \\
\hline & SI & 103 & 38 & & & \\
\hline \multirow{3}{*}{ Trabaja fuera de la casa } & $\mathrm{NO}$ & 136 & 119 & \multirow{3}{*}{1,47} & \multirow{3}{*}{$1,01-2,1$} & \multirow{3}{*}{0,0269} \\
\hline & & & & & & \\
\hline & SI & 82 & 106 & & & \\
\hline
\end{tabular}

Fuente: base de datos.

\section{DISCUSIÓN}

El hecho más relevante de este análisis tiene que ver con la concordancia encontrada en la prevalencia de factores de riesgo para el cáncer reportada por la literatura y los hallazgos evidenciados en el desarrollo de este estudio, circunstancia preocupante en la medida en que muchos de estos riesgos también se encuentran asociados con la renuencia a practicar el AEM.

La incidencia del cáncer de mama en los Estados Unidos ha disminuido significativamente en los últimos años, probablemente por los cambios realizados en las tecnologías diagnósticas, al incrementarse la cobertura de mujeres con mamografías, lo que permite diagnósticos más tempranos, en primer lugar, y también por la interrupción del uso terapéutico de una combinación de estrógeno más progestina en mujeres postmenopáusicas, para la prevención primaria de enfermedades crónicas, al comprobarse que los riesgos excedían a los beneficios ${ }^{(13)}$. Sin embargo, en muchos países en vía de desarrollo, y específicamente en países de Latinoamérica y el Caribe, se observa un patrón contrario con un incremento paulatino en la mortalidad, mayor que en los países desarrollados, lo que indica probablemente deficiencias en el tratamiento y en la implementación de estrategias de detección temprana y en la promoción de salud y prevención específica de esta patología ${ }^{(14)}$.

En Colombia, la mortalidad por cáncer de mama muestra una clara tendencia al incremento en la última década ${ }^{(2)}$. Para el año 2000 , el cáncer de mama ocupó el tercer lugar como causa de muerte por cáncer entre mujeres, con 1.542 muertes registradas, después del cáncer de estómago y el de cuello uterino ${ }^{(2)}$. En el país no se dispone de información sobre los estados en que se diagnostica la enfermedad; los datos de las pacientes atendidas en año 2004, en el Instituto Nacional de Cancerología (INC), muestran que alrededor del 64\% de los casos nuevos llegan en estadios avanzados de II B y más ${ }^{(3)}$.

En el Hospital de Ginecología del Instituto Mexicano del Seguro Social, se reporta en un seguimiento de 1991 a 1995, que el grupo más afectado por cáncer mamario es el de 40 a 
50 años, teniendo como factores de riesgo agregado la no lactancia y la obesidad 18). También se mencionan, en otros estudios, antecedente familiar de cáncer de mama, enfermedad benigna proliferativa de la mama, edad mayor a 40 años, menarquía antes de los 12 años, menopausia después de los 55 años, nuliparidad, primer embarazo en mayores de 35 años, escaso número de hijos y lactancia corta o artificial, ingesta de estrógenos mayor de 5 años y exposición a radiación ionizante ${ }^{(15,16)}$.

Por otra parte, Doris Ortega ${ }^{(17)}$, hace una relación de los factores reproductivos relacionados con el cáncer de mama, entre los que destaca menarquía a edad temprana, nueve años; menopausia a edad tardía, 55 años o más; nacimiento del primer hijo a edad superior a los 30 años; uso durante la menopausia de terapia hormonal de reemplazo, por periodos superiores a tres años, y ausencia de lactación o lactar por periodos muy cortos y con baja intensidad.

Como factores ambientales se ha descrito exposición a agroquímicos, productos químicos o contaminantes. En relación con la alimentación, consumo de dos o más copas / día de alcohol obtenido de granos, alimentos ricos en grasas animales y saturadas, y escaso consumo de alimentos con alto contenido de fibra y vitaminas A y C. Últimamente, se ha reportado como riesgo, la ingesta de phthates, compuesto químico liberado por plásticos en los que se ha depositado agua o alimentos ${ }^{(18)}$. El sedentarismo se ha encontrado asociado con la presencia del cáncer mamario.

El cáncer de mama puede ser limitado, e incluso curado, cuando se diagnostica en las etapas I y II; con mucha menos probabilidad en etapas tardías como III y IV. Cualquiera sea la etapa del diagnóstico, es posible alguna forma de tratamiento, pero los resultados difieren en cada caso, en función de las condiciones generales de salud y la edad de la mujer.

Los tres métodos de vigilancia más eficaces para el diagnóstico temprano del cáncer de mama, autoexamen, examen clínico y mamografía han demostrado claramente su eficacia, al disminuir la mortalidad hasta en un $30 \%$, en varios países. Esto en razón a que la historia natural del cáncer de seno permite que esta entidad pueda ser reconocida tempranamente, lo que ha valorizado enormemente las campañas de detección temprana como herramientas básicas en la disminución de la mortalidad ${ }^{(19-21)}$.

La limitación principal para las técnicas del autoexamen es la dificultad intrínseca para la palpación de las glándulas mamarias. A pesar de que se disponga de una capacitación efectiva, la sensibilidad de la palpación como elemento básico de las técnicas del autoexamen, depende del factor humano, es decir, de la rigurosidad y precisión con la que se lleve a cabo.

En el autoexamen es fundamental la capacidad y la confianza de la mujer en sus propias habilidades: en la de tipo manual para palpar, y en la intelectual y emocional para interpretar una pequeña masa en el seno o la axila como algo normal y transitorio, o como algo que requiere la intervención de un profesional de la salud ${ }^{(22)}$.

De acuerdo con los resultados de la Encuesta Nacional de Salud Reproductiva, adelantada en México en 2003 , 74\% de las mujeres revisa sus mamas y un porcentaje menor (37.4\%) acude al personal de salud para la revisión clínica de éstas. Las principales características vinculadas con la revisión de los senos fueron la edad, el lugar de residencia, la escolaridad y el nivel socioeconómico. Dicha revisión fue significativamente mayor en las mujeres residentes de áreas urbanas respecto de las residentes de áreas rurales. Asimismo, se observó un incremento significativo de esta práctica conforme aumentó la edad, la 
escolaridad y el nivel socioeconómico de las entrevistadas. Los motivos mencionados con más frecuencia por las mujeres que no practican el AEM son: no consideran que sea una práctica importante 0 no saben cómo realizarla. Las razones anteriores no variaron significativamente de acuerdo con el nivel de escolaridad de las entrevistadas.

También se encontró en la misma encuesta que las mujeres con escolaridad de al menos preparatoria llevan a cabo esta práctica seis veces más frecuentemente que las mujeres sin estudios ${ }^{(23)}$.

En las entidades sanitarias, ha hecho carrera como estrategia para promover la participación de las mujeres en programas de control y detección temprana del cáncer de mama la distribución de material educativo impreso y el desarrollo de conferencias. Todo parece indicar que el impacto de estas campañas se limita a engrosar la documentación institucional, pues aparte del registro de informes que relacionan el número de ejemplares distribuidos o el volumen de usuarias intervenidas, sin que ello repercuta significativamente en los índices de detección temprana de los casos de cáncer mamario, estos informes se entremezclan con las actividades de otros programas limitando la posibilidad de analizar objetivamente la eficacia de las metodologías educativas.

Por otra parte, en Colombia, existen marcadas diferencias entre el plan obligatorio de salud del régimen subsidiado, que tiene una cobertura restringida en servicios especializados y hospitalarios con respecto al Plan Obligatorio de Salud del Régimen Contributivo y por él se paga a los aseguradores aproximadamente la mitad del per cápita que el sistema reconoce por una afiliado al Régimen Contributivo. Recientemente se ha extendido la cobertura con un plan aún más reducido, que equivale aproximadamente a un cuarto del seguro completo del Contributivo, en términos de costo y cobertura ${ }^{(24)}$. Esto significa que en términos del tipo de atención, existen claras diferencias en la accesibilidad y calidad de servicios, en función del sistema de afiliación.

En el diseño de programas de tamizaje y protocolos de manejo resulta fundamental tener en cuenta las evidencias y factores determinantes para que las acciones emprendidas sean efectivas, particularmente entre mujeres con limitaciones de acceso a los servicios de salud. La precariedad en las coberturas y motivación invita a echar mano de estrategias como la llamada comunicación persuasiva. La salud, precisa de metodologías de comunicación que consigan obtener las metas de un mensaje convincente, ya que este depende de los abordajes y apelativos diferenciados relacionados a los objetivos, lenguaje y sujeto receptor (25).

El proceso de persuasión ocurre en cinco etapas: un mensaje es enviado a un receptor; el mensaje es percibido e identificado por el receptor; el intérprete (receptor) atribuye un significado al mensaje; el significado dentro del receptor actúa como un estímulo para cualquier efecto que pueda ocurrir; y los efectos en el receptor generan una acción que puede relacionarse con la meta deseada por el agente de salud. El concepto importante, central en este modelo, es que el estímulo para el cambio es el significado suscitado en el receptor ${ }^{(26)}$.

En la medida en que la comunicación persuasiva es útil para con los receptores, puede ser utilizada como dinámica para facilitar el diagnóstico precoz y disminuir el riesgo de cáncer en las mujeres, esto es, el uso de este proceso puede generar comportamientos preventivos. Algunos estudios muestran que al utilizar campañas publicitarias dirigidas al público deseado, con la utilización de lenguaje apropiada a una determinada población, el suceso fue observado ${ }^{(27,28)}$. 


\section{CONCLUSIÓN}

Los principales factores asociados con la no realización del AEM en la población estudiada se relacionan con aspectos predominantemente sociales, culturales y económicos; la mayoría susceptibles de modificación si se readecuan estrategias y políticas en lo que tiene que ver con el diseño y planeación de los programas de promoción y prevención específicos.

\section{REFERENCIAS}

1. Carlson RW, Anderson BO, Burstein HJ, Carter WB, Edge SB, Farrar WB, et al. Invasive breast cancer. Journal of the National Comprehensive Cancer Network: JNCCN. 2007;5(3):246.

2. Piñeros M, Murillo R. Incidencia de cáncer en Colombia: importancia de las fuentes de información en la obtención de cifras estimativas. Revista Colombiana de Cancerología. 2004;8(1):5-14.

3. Ministerio de Protección Social, Instituto Nacional de Cancerología, editors. Anuario estadístico 2004. Bogotá: Ministerio de Protección Socia; 2005.

4. Ferlay J, Bray F, Pisani P, Parkin DM. GLOBOCAN 2002: Cancer Incidence, Mortality and Prevalence Worldwide IARC Cancer Base No. 5. version 2.0. Lyon: IARC press; 2004. 5. Henderson IC, Pike MC, Bernstein L, Ross RK. Cáncer de mama. In: Murphy G, Lawrence WJ, Lenhard RJ, editors. Oncologia Clinica-Manual de la American Cancer Society. 2a ed. Washington: Organización Panamericana de la Salud; 1996. p. 224-48. 6. Wiesner C. Determinantes psicológicos, clínicos y sociales del diagnóstico temprano del cáncer de mama en Bogotá, Colombia. Rev Colomb Cancerol. 2007;11(1):13-22.

7. Schwartsmann G. Breast cancer in South America: challenges to improve early detection and medical management of a public health problem. Journal of Clinical Oncology. 2001;19(Supl 18):118s-24s.

8. Ramirez AJ, Westcombe AM, Burgess CC, Sutton S, Littlejohns P, Richards MA. Factors predicting delayed presentation of symptomatic breast cancer: a systematic review. Lancet. 1999;353(9159):1127-31.

9. Ghafoor A, Jemal A, Ward E, Cokkinides V, Smith R, Thun M. Trends in breast cancer by race and ethnicity. CA Cancer J Clin. 2003;53(6):342-55.

10. Lannin DR, Mathews HF, Mitchell J, Swanson MS, Swanson FH, Edwards MS. Influence of socioeconomic and cultural factors on racial differences in late-stage presentation of breast cancer. JAMA. 1998;279(22):1801-7.

11. Wagle A, Komorita NI, Lu ZJ. Social support and breast self-examination. Cancer nursing. 1997;20(1):42.

12. Alcaldia Mayor de Tunja. Plan de Ordenamiento Territorial (POT). In: Planeación Od, editor. Tunja: Alcaldia Mayor de Tunja; 2005. p. 520.

13. Rossouw JE, Anderson GL, Prentice RL, LaCroix AZ, Kooperberg C, Stefanick ML, et al. Writing Group for the Women's Health Initiative Investigators. Risks and benefits of estrogen plus progestin in healthy postmenopausal women: principal results from the Women's Health Initiative randomized controlled trial. JAMA. 2002;288(3):321-33.

14. Robles SC, Galanis E. Breast cancer in Latin America and the Caribbean. Revista Panamericana de Salud Pública. 2002;11(3):178-85.

15. Heredia MA, Bautista-Samperio L, Pérez RTA. Correlación de factores de riesgo y hallazgos clínicos para cáncer mamario en pre y posmenopáusicas. Rev Fac Med UNAM. 2007;50(3):110-4.

16. Vogel VG. Breast cancer in younger women: Assessment and risk The Female Patient. 1999;24:81-6 
17. Ortega D. Diagnóstico tardío del cáncer de mama en México: desencuentro entre las mujeres y los servicios de salud. In: Bronfman M, Denman C, editors. salud reproductiva. México: Instituto Nacional de Salud Pública; 2003. p. 157-82.

18. Brewster A, Helzlsouer K. Breast cancer epidemiology, prevention, and early detection Current Opinion in Oncology. 2001;13(6):420-5.

19. Hill D, White V, Jolley D, Mapperson K. Self examination of the breast: is it beneficial? Meta-analysis of studies investigating breast self examination and extent of disease in patients with breast cancer. British Medical Journal. 1988;297(6643):271-5.

20. Poblano-Verástegui O, Figueroa-Perea JG, López-Carrillo L. Condicionantes institucionales que influyen en la utilización del examen clínico de mama. Salud Pública de México. 2004;46(4):294-305.

21. Ruisánchez P N, Álvarez MT, Menéndez A I, Ramírez B I. Alternativas preventivas en cáncer de mama. Rev Inst Nal Cancerol (Mex). 2000;46(4):232-6.

22. Gifford SM. The meaning of lumps: a case study of the ambiguities of risk. In: Janes CR, Stall R, Gifford SM, editors. Anthropology and epidemiology: Interdisciplinary approaches to the study of health and disease. Dordrecht: Reidel Publishing Company; 1986. p. 213-46.

23. López Carrillo L, Suárez-López L, Torres-Sánchez L. Detección del cáncer de mama en México: síntesis de los resultados de la Encuesta Nacional de Salud Reproductiva. Salud Pública de México. 2009;51(Supl 2):s345-s9.

24. Martinez Martin F. ¿Subsidios o Seguridad Social? El no pago de la porción social del salario. Journal [serial on the Internet]. 2005 Date: Available from:

http://www.saludcolombia.com/actual/documentos/El\%20no\%20pago\%20de\%20la\%20porcio n\%20social\%20del\%20salario.pdf.

25. Tonani M, Carvalho EC. Cancer risk and preventive behavior: persuasion as an intervention strategy. Revista Latino-Americana de Enfermagem. 2008;16(5):864-70.

26. Littlejohn SW. Fundamentos teóricos da comunicação humana. Rio de Janeiro:

Guanabara Koogan; 2005.

27. Cohen L, Dobson H, McGuire F. Promoting breast screening in Glasgow. Health Bulletin. 2000;58(2):127.

28. Ward B, Bertera EM, Hoge P. Developing and evaluating a Spanish TEL-MED message on breast cancer. Journal of Community Health. 1997;22(2):127-35.

ISSN 1695-6141

(c) COPYRIGHT Servicio de Publicaciones - Universidad de Murcia 